\title{
Uniqueness of the Infinite Cluster and Continuity of Connectivity Functions for Short and Long Range Percolation
}

\author{
M. Aizenman ${ }^{1, \star}$, H. Kesten ${ }^{2, \star \star}$ and C. M. Newman ${ }^{3, \star \star \star}$ \\ 1 Departments of Mathematics and Physics, Rutgers University, New Brunswick, New Jersey 08903, \\ USA \\ 2 Department of Mathematics, Cornell University, Ithaca, New York 14853 USA and Institute for \\ Mathematics and its Applications, University of Minnesota, Minneapolis, Minnesota 55455, USA \\ 3 Department of Mathematics, University of Arizona, Tucson, Arizona 85721, USA
}

\begin{abstract}
For independent translation-invariant irreducible percolation models, it is proved that the infinite cluster, when it exists, must be unique. The proof is based on the convexity (or almost convexity) and differentiability of the mean number of clusters per site, which is the percolation analogue of the free energy. The analysis applies to both site and bond models in arbitrary dimension, including long range bond percolation. In particular, uniqueness is valid at the critical point of one-dimensional $1 /|x-y|^{2}$ models in spite of the discontinuity of the percolation density there. Corollaries of uniqueness and its proof are continuity of the connectivity functions and (except possibly at the critical point) of the percolation density. Related to differentiability of the free energy are inequalities which bound the "specific heat" critical exponent $\alpha$ in terms of the mean cluster size exponent $\gamma$ and the critical cluster size distribution exponent $\delta$; e.g., $1+\alpha \leqq \gamma(\delta / 2-1) /(\delta-1)$.
\end{abstract}

\section{Introduction}

In this paper, we present general results dealing with three related issues in percolation theory: a) uniqueness of the infinite cluster, b) continuity (in the natural parameters) of the connectivity functions and c) continuous differentiability of the "free energy" function. New relations are derived here between these three properties and used for the resolution of some old problems in the mathematical analysis of percolation. Specifically, the question of uniqueness of the infinite cluster is resolved (for independent, translation invariant models on finite dimensional lattices) by first proving uniqueness to be equivalent to either of the continuity statements b) or c)

* Research supported in part by NSF Grant PHY-8605164

$\star \star$ Research supported in part by the NSF through a grant to Cornell University

$\star \star \star$ Research supported in part by NSF Grant DMS-8514834 
and then establishing differentiability c). The equivalence between these three issues is quite similar to the situation in Ising models, where differentiability of the free energy is equivalent to continuity of the energy density and either of these implies the absense of any translation invariant ergodic Gibbs distributions other than the usual ones given by plus or minus boundary conditions [Le].

In addition to these results, extensions of property c) are obtained which give d) sufficient conditions for twice-differentiability of the free energy and related critical exponent inequalities. (These are presented in Sect. 3 below following the proof of Proposition 1.5.)

a. Motivation. The motivation for studying uniqueness of infinite clusters is multifold:

i) The qualitative properties of the percolating phase are of intrinsic interest. It was shown in [NS1] that for a large class of both independent and dependent models, nonuniqueness of infinite clusters cannot occur unless there are (with probability one) infinitely many distinct infinite clusters. However, nonuniqueness has not been shown to occur in any natural nontrivial model (it does of course occur in independent percolation on a Bethe lattice).

ii) Uniqueness is a rather usefull property for the development of rigorous renormalization arguments, both for short range [ACCFR] and long range[NS2] models.

iii) The uniqueness and intersection properties of "incipient infinite clusters" [Co; K2] at critical points seem to be closely connected with the values of critical exponents. For example, nonuniqueness (of a certain sort) implies mean field values for at least some exponents [AN1, Sect. 3.2] while uniqueness appears related to hyperscaling relations [Co; K3]. Some results have been obtained concerning uniqueness of two-dimensional incipient infinite clusters [K2] and related objects [CCN, Appendix] but little is known in general. The results of this paper concern uniqueness of infinite clusters away from a critical point or at a critical point with strictly positive percolation density. They may be regarded as a first step in preparation for a rigorous analysis of the uniqueness/intersection properties of incipient infinite clusters.

iv) There are close relations between uniqueness of the infinite cluster and qualitative properties of thermodynamic functions. Such a link appeared first in the result of van den Berg and Keane [BK] that uniqueness implies continuity of the percolation density except possibly at the critical point. The analysis presented here adds to our understanding of such relations in two ways: a) Our general uniqueness result applies also to the critical point of the long-range one dimensional models with bond occupation probabilities asymptotic to $\beta /|x-y|^{2}$ [NS2], whose percolation density is known to be discontinuous at the critical point [AN2]. Thus the restriction in the result of $[\mathrm{BK}]$ to points other than the critical point is indeed necessary. b) In this paper we introduce another such relation, namely the threefold equivalence of the uniqueness of the infinite cluster, continuity of the connectivity functions, and differentiability of the free energy (the mean number of clusters per site). This relation involves no caveats about the critical point.

We next introduce the setup for our main results. At the end of the introduction, we discuss some of the previously known results on uniqueness. 
b. Setup. In Sects. 1-4, we restrict attention to independent, translation-invariant bond percolation models in which an occupied (or passable) bond may occur between any pair of sites $\{x, y\}$ in $\mathbb{Z}^{d}, d \geqq 1$. Such a model may be thought of as defined by independent occupation variables $\left\{n_{\{x, y\}}: x, y \in \mathbb{Z}^{d}, x \neq y\right\}$ taking the values 1 (bond occupied) or 0 (bond vacant) with

$$
\operatorname{Prob}\left(n_{\{x, y\}}=1\right)=p_{y-x}=1-\exp \left(-\beta J_{y-x}\right),
$$

where $0 \leqq p_{y-x}<1, \beta>0$ and $J_{y-x}=J_{x-y} \geqq 0$. We sometimes exclude $p_{z}=1$ (or $\beta J_{z}=\infty$ ) to avoid the extra considerations needed for this degenerate case. Ordinary nearest neighbor bond percolation corresponds to the choice of $p_{y-x}=p$ (or $J_{y-x}=1$ ) for $y$ and $x$ nearest neighbors and $p_{y-x}=0$ (or $J_{y-x}=0$ ) otherwise. The generalization of our results to lattices other than the hypercubic $\mathbb{Z}^{d}$ is straightforward; the generalization to site percolation will be discussed in Sect. 5 below. A generalization to directed percolation models has not been made.

A cluster $C$ in such a model consists of a maximal set of sites which are all connected by paths of occupied bonds. The percolation density $P_{\infty}$ is the probability that $C(0)$, the cluster containing the origin, is infinite. When $P_{\infty}=0$, there are with probability one no infinite clusters (although one may still be able to define incipient infinite clusters [K2]).

The (two-point) connectivity function $\tau(x, y)$ is the probability that $x$ and $y$ belong to the same cluster. The $n$-point connectivity function, $\tau\left(x_{1}, \ldots, x_{n}\right)$ is similarly the probability that $x_{1}, \ldots, x_{n}$ all belong to the same cluster. We will call a model irreducible if $\tau(x, y)>0$ for all $x, y$; i.e., if $\mathbb{Z}^{d}$ cannot be partitioned into two nonempty subsets, $\mathbb{L}_{1}$ and $\mathbb{L}_{2}$, with $p_{y-x}=0$ whenever $x \in \mathbb{L}_{1}$ and $y \in \mathbb{L}_{2}$. Clearly, reducible models can have distinct infinite clusters.

c. Main Results. Following are the statements of our main results for independent $d$-dimensional (short or long range) bond percolation models.

Proposition 1.1. In any irreducible, translation invariant model: if $P_{\infty}>0$, then there is exactly one infinite cluster (with probability one).

Remark. When $\sum_{x} p_{x}=\infty$ (or $\sum_{x} J_{x}=\infty$ ), it is immediate that $P_{\infty}=1$, since, with probability one, each site has infinitely many occupied bonds incident to it. It has further been proved in [GKM] that for irreducible such models, $\tau(x, y)=1$ for all $x$ and $y$; i.e. all sites in $\mathbb{Z}^{d}$ are connected. This result can alternatively be obtained as an immediate corollary of Proposition 1.1; we note in this regard that the proof of Proposition 1.1 for such models is much shorter than for the general case (see the fourth remark following Proposition 1.5 below).

The next two propositions refer to continuity properties of percolation models. It is often convenient to focus on one parameter families of models with $P_{\infty}, \tau(x, y)$ etc. regarded as functions of that one parameter, with all other parameters held fixed. One natural choice is to take $\beta$ as the varying parameter with all the $J_{y-x}$ 's fixed. Another choice is to pick some finite set $\Gamma$ of nonzero $z$ 's in $\mathbb{Z}^{d}$, which is symmetric with respect to reflection through the origin, and consider the models with $p_{y-x}=p$ for each $x$ and $y$ such that $y-x$ is in $\Gamma$; here $p$ is the varying parameter and all other $p_{y-x}$ 's are fixed. For example, in order to study the dependence of a model on a single 
$p_{z}, \Gamma$ would be chosen to be $\{z,-z\}$; on the other hand, in standard nearest neighbor models on $\mathbb{Z}^{d}, \Gamma$ would consist of the $2 d$ neighbors of the origin and all other $p_{y-x}$ 's would be fixed at zero. The critical value $p_{c}$ or $\beta_{c}$ is defined as the smallest value of $p$ or $\beta$ above which $P_{\infty}>0$; i.e.

$$
\begin{aligned}
& p_{c}=\inf \left\{p \in(0,1]: P_{\infty}(p)>0\right\}, \\
& \beta_{c}=\inf \left\{\beta \in(0, \infty]: P_{\infty}(\beta)>0\right\} .
\end{aligned}
$$

It is easy to see that $\beta_{c}=0$ if (and only if) $\sum_{z} J_{z}=\infty$.

In spite of the convenience of the one parameter models, it is instructive to bear in mind that the complete parameter set of bond percolation models, $\left\{p_{z}\right\}$, is infinite in number. It is therefore appropriate to introduce a notion of convergence which allows all the parameters to vary independently. We say that a function $F$ (of the $p_{z}$ 's) is continuous in the $L^{1}$ sense at $\left\{\bar{p}_{z}\right\}$ if $F\left(\left\{p_{z}\right\}\right) \rightarrow F\left(\left\{\bar{p}_{z}\right\}\right)$ whenever $\sum_{z}\left|p_{z}-\bar{p}_{z}\right| \rightarrow 0$. (Note that we allow $\sum_{z} \bar{p}_{z}=\infty$ in this definition.)

To see that this type of continuity is natural, consider the special case where $F$ is the probability that no occupied bond is incident to the origin. Then $F=\prod_{z}\left(1-p_{z}\right)$, which is easily seen to be continuous in the $L^{1}$ sense. Similarly, the probability that the cluster of a given site is a specified finite set is $L^{1}$-continuous. Note also that for a one parameter ( $p$ or $\beta$ ) model, continuity in the $L^{1}$ sense implies continuity in $p$ or (if $\left.\sum_{z} J_{z}<\infty\right)$ in $\beta$.

Proposition 1.2. Let $\left\{\bar{p}_{z}\right\}$ denote the parameter set of some irreducible, translation invariant model. Then for any fixed $n$ and fixed $x_{1}, \ldots, x_{n}, \tau\left(x_{1}, \ldots, x_{n}\right)$, considered as $a$ function of all the $p_{z}$ 's, is continuous in the $L^{1}$ sense at $\left\{\bar{p}_{z}\right\}$. In particular in an irreducible, one parameter ( $p$ or $\beta$ ) model, the connectivity functions depend continuously on $p($ in $(0,1])$ or $\beta$ (in $(0, \infty])$.

Remark. A lack of continuity at $p=0$ can only happen when the $p=0$ model is reducible. This occurs, for example, in a three dimensional nearest neighbor model in which $p=p_{(0,0,1)}$ while $p_{(1,0,0)}$ and $p_{(0,1,0)}$ remain fixed above the two dimensional percolation threshold; here if $x_{1}$ and $x_{2}$ are in different two dimensional layers, then $\tau\left(x_{1}, x_{2}\right)$ vanishes at $p=0$ but as $p \rightarrow 0$, it tends to the square of the two dimensional percolation density. A lack of continuity at $\beta=0$ occurs only when $\sum J_{z}=\infty$, in which case $\tau\left(x_{1}, \ldots, x_{n}\right)=1$ for $\beta>0$ but vanishes for $\beta=0$.

One may appreciate the continuity result for connectivity functions more after noticing that the percolation density $P_{\infty}$ is not, in general, continuous in the $L^{1}$ sense. That fact is clearly seen in the situation where the percolation transition is driven by the long bonds, i.e. in one dimension. Any one dimensional model with $\sum p_{z}<\infty$ and $P_{\infty}>0$ (see [NS2]) is a limit in the $L^{1}$ sense, of finite range models for which $P_{\infty}=0$. (On the other hand, it may very well be the case that $P_{\infty}$ is continuous, in the $L^{1}$ sense, at the parameter set $\left\{\bar{p}_{z}\right\}$ of any irreducible, translation invariant model in more than one dimension.)

An even more drastic discontinuity of $P_{\infty}$ occurs in one dimensional "borderline" models, with $J_{x-y} \sim 1 /|x-y|^{2}$. At the critical point of such a model, $P_{\infty}$ is 
discontinuous even as a function of finitely many $p_{z}$ 's with all others held fixed. This follows from the fact [AN2] that $P_{\infty}$ either vanishes or else is bounded below by $\left[\lim \sup z^{2} p_{z}\right]^{-1 / 2}$, a bound which is unaffected by changing finitely many $p_{z}$ 's. To construct a one parameter model with such a discontinuity in $P_{\infty}$, take any $\left\{\bar{p}_{z}\right\}$ with $\lim z^{2} \bar{p}_{z}$ in $(1, \infty)$. Then the one parameter model with $p_{z}=p$ for $|z| \leqq R$ and $p_{z}=\bar{p}_{z}$ for $|z|>R$ will have $p_{c}$ neither zero (if $R$ is chosen so that $\sum_{|z|>R} \bar{p}_{z}<1$ ) nor one [NS2] but will have a discontinuity in $P_{\infty}(p)$ at $p=p_{c}$ as just explained.

The next proposition shows that a discontinuity of $P_{\infty}$ like the one exhibited in the previous paragraph, can occur only at a critical point. This result is actually a rather direct corollary (as explained in the latter part of Sect. 4 below) of the uniqueness proven in Proposition 1.1 and of the earlier result (and method) of van den Berg and Keane [BK].

Proposition 1.3. In any irreducible, translation invariant, one parameter ( $p$ or $\beta$ ) model, $P_{\infty}$ is a continuous function of $p$ in $(0,1]$ or $\beta$ in $(0, \infty]$ except possibly at $p=p_{c}$ or $\beta=\beta_{c}$.

Remark. Although $P_{\infty}$ is discontinuous at the critical point of one dimensional $1 /|x-y|^{2}$ models [AN2], it is expected to be continuous for most other models. Unfortunately, even for standard nearest neighbor models, this has not yet been proven except for $d=2$ [Rus]. However, there exist some sufficient conditions for continuity of $P_{\infty}$ at the critical point [AB2; N2]. One of these [AB2] is the validity of the "triangle criterion" (first introduced in $[\mathrm{AN} 1]$ ); i.e. a bound on

$$
\sum_{x} \sum_{y} \tau(0, x) \tau(x, y) \tau(y, 0)
$$

which is uniform for $\beta<\beta_{c}$ (we assume here that $0<\beta_{c}<\infty$ ). The triangle criterion, which also implies that certain critical exponents take on their mean field values [AN1; $\mathrm{AB} 2$ ], is only expected to be valid for $d>6$ (in short range models); it is thus an overly strong condition for continuity of $P_{\infty}$. However, the uniqueness result of Proposition 1.1 implies much weaker conditions which would guarantee that $P_{\infty}$ vanishes (and hence is continuous) at the critical point: namely any a priori bound which would force $\tau(x, y)$ to become small as $|x-y| \rightarrow \infty$, uniformly in $\beta<\beta_{c}$. This is so, because if $P_{\infty}\left(\beta_{c}\right)>0$ (and the model is irreducible at $\beta_{c}$ ), then for all $x, y$,

$$
\begin{aligned}
\lim _{\beta \uparrow \beta_{c}} \tau(x, y) & =\operatorname{Prob}_{c}(x \text { and } y \text { belong to the same cluster }) \\
& \geqq \operatorname{Prob}_{c}(C(x) \text { and } C(y) \text { are infinite }) \\
& \geqq\left(P_{\infty}\left(\beta_{c}\right)\right)^{2}>0,
\end{aligned}
$$

where the subscript $c$ denotes evaluation at $\beta=\beta_{c}$. Here the first equality is a consequence of left continuity of the connectivity function (which does not require Proposition 1.2), the first inequality is due to uniqueness of the infinite cluster and the second follows from the Harris-FKG inequalities [Har; FKG]. An example of such a sufficient condition is the uniform boundedness, as $\beta \uparrow \beta_{c}$, of

$$
\sum_{x}[\tau(0, x)]^{m} /\|x\|^{d}
$$


for some finite $m$, where $\|x\|$ is the Euclidean length of $x$ in $\mathbb{Z}^{d}$. We note that the relation, discussed in this remark, between uniqueness, continuity of $P_{\infty}$ and decay as $|x-y| \rightarrow \infty$ of $\tau(x, y)$ (uniform in $\beta<\beta_{c}$ ) has an analogue for general Ising systems [BL].

The methods we use to obtain the uniqueness and continuity results of Propositions 1.1 and 1.2 seem to us to be of independent interest. They show the applicability of arguments which have been developed in other areas of Statistical Mechanics (see e.g. [Le]) but have not been much employed in the study of percolation.

A key quantity in our analysis is

$$
f=\sum_{n=1}^{\infty} \frac{1}{n} P_{n}=E\left(|C(0)|^{-1}\right),
$$

where $|C(0)|$ denotes the number of sites in the cluster $C(0)$ and

$$
P_{n}=\operatorname{Prob}(|C(0)|=n) \text {. }
$$

Note that even though we deal with bond percolation, the $n$ in $P_{n}$ refers to the number of sites in the cluster. $f$ represents the mean number of clusters per site and was first exploited as such in [SE]. On the other hand, $f$ may also be regarded as the percolation analogue of the free energy in Ising or Potts models [KF;FK]. Propositions 1.1 and 1.2 are direct consequences (as demonstrated in Sect. 4 below) of the following two propositions about the dependence of the free energy $f$ on $p$ or $\beta$ in the one parameter models.

Proposition 1.4. In any translation invariant bond percolation model:

i) $f$ is a convex function of $p$ whose one-sided derivatives are

$$
\begin{aligned}
& \frac{\partial f}{\partial p}(p-0)=-[2(1-p)]^{-1} \sum_{x \in \Gamma}(1-\tau(0, x)), \text { for } 0<p<1, \\
& \frac{\partial f}{\partial p}(p+0)=-[2(1-p)]^{-1} \sum_{x \in \Gamma}\left(1-\tau^{+}(0, x)\right), \text { for } 0 \leqq p<1,
\end{aligned}
$$

where

$$
\tau^{+}(x, y)=\tau(x, y)+\operatorname{Prob}(x \text { and } y \text { belong to distinct infinite clusters) }
$$

Likewise,

ii) $f$ is a convex function of $\beta$ with the one-sided derivatives

$$
\begin{aligned}
& \frac{\partial f}{\partial \beta}(\beta-0)=-(1 / 2) \sum_{x \in \mathbb{Z}^{d}} J_{x}(1-\tau(0, x)), \text { for } \beta>0, \\
& \frac{\partial f}{\partial \beta}(\beta+0)=-(1 / 2) \sum_{x \in \mathbb{Z}^{d}} J_{x}\left(1-\tau^{+}(0, x)\right), \text { for } \beta>0 .
\end{aligned}
$$

Thus

iii) $\partial f / \partial p$ has a discontinuity at some $p$ in $(0,1)$ if and only if for some $x$ in $\Gamma$,

$$
\text { Prob }(0 \text { and } x \text { belong to distinct infinite clusters })>0
$$


at that value of $p$. Similarly $\partial f / \partial \beta$ has a discontinuity at some $\beta$ in $(0, \infty)$ if and only if for some $x$ with $J_{x}>0,(1.10)$ is valid (or equivalently $\partial f / \partial p_{x}$ is discontinuous) at that value of $\beta$.

Proposition 1.5. In any translation invariant model, $f$ is a continuously differentiable function of $p$ in $(0,1)$. Thus (by the last statement of Proposition 1.4) $f$ is also a continuously differentiable function of $\beta$ in $(0, \infty)$.

Remarks. i) The proof that $f$ is differentiable (Proposition 1.5) leads to bounds on the second and higher derivatives of $f$ which in turn imply various inequalities for the critical exponents $\alpha, \gamma$ and $\delta$ (see (3.22)-(3.27) below). Precise statements of these extensions of Proposition 1.5 are given in Proposition 3.3 and its corollaries.

ii) A proof of Proposition 1.5 is implicitly contained in the proof of [K1;p. 252] that $f$ is twice continuously differentiable in standard two-dimensional models. Section 3 below presents that proof, which is based on a lattice animal expansion, explicitly. The first use of the lattice animal representation to study the differentiability of $f$ appears to be in [G2].

iii) The proof that $f$ is differentiable is independent of Proposition 1.4 and its proof (which is given in Sect. 2 below). It was pointed out to us that one can therefore use the differentiability of $f$ and standard convex function arguments (cf. [R], Theorem 25.7) to simplify both the statement and proof of Proposition 1.4 by eliminating the need to explicitly consider one-sided derivatives. We have kept the present structure for two related reasons. First, it emphasizes the three fold equivalence between uniqueness of the infinite cluster, continuity of $\tau(0, x)$ and differentiability of $f$. Second, the a priori expressions for the one-sided derivatives of $f$ are the natural analogues for the corresponding expressions in Ising systems [Le]; such analogies between percolation and Ising models can be helpful for both subjects.

iv) For models in which $\sum_{x} p_{x}=\infty, f \equiv 0$ since $P_{\infty} \equiv 1$ by the remark following Proposition 1.1 above. Since the derivative formula (1.5) is applicable even in that situation, it implies that $\tau(x, y)=1$ for all $x, y$ with $p_{y-x}>0$. Thus a proof of the [GKM] total connectivity result for irreducible such models can be based on (1.5), and the result is a particular case of Proposition 1.1.

v) The convexity of $f$ seems not to have been noted previously, perhaps because the analogous quantity in site percolation is not convex. However, as already pointed out in [DN, Sect. 3], the site percolation free energy may be made convex by the addition of an explicit polynomial in $p$. All our results can be extended, with suitable adjustments, to site percolation as we explain below in Sect. 5 .

vi) The usefulness of convexity and related properties of $f(\beta)$ extends to

$$
f(\beta, h)=\sum_{n=1}^{\infty} \frac{1}{n} P_{n} e^{-n h}
$$

as can be seen in the methods and results of $[\mathrm{AB} 1]$.

Before concluding this introduction, let us briefly review previous results on uniqueness of infinite clusters. Previous proofs of uniqueness were confined to site percolation or nearest neighbor bond percolation on $\mathbb{Z}^{d}$ or other $d$-dimensional 
lattices. Uniqueness was known for $d=2$ and any $p>p_{c}$ [Har; F] but for $d>2$ it was only known for $p>p_{c}^{\prime}$ [ACCFR], where $p_{c}^{\prime}$ is a limit of "slab thresholds" conjectured, but not yet proven, to coincide with $p_{c}$.

There was a nonrigorous but heuristically appealing argument [Ki] which supported uniqueness for general $d$ and $p$ on the grounds that two potentially distinct infinite clusters should infinitely often come close to each other and thus should have zero probability of remaining distinct. In a sense, the main result of [NS1] was a rigorous version of that argument (applicable also to general bond models and to a large class of dependent percolation models) which ruled out nonuniqueness via any finite number of distinct infinite clusters. The work of [NS1] suggests that it would be hard to rule out the coexistence of infinitely many distinct infinite clusters by such an argument alone. Of course, infinitely many distinct infinite clusters do occur in Bethe lattice models. It is an instructive exercise, which we leave for the reader, to discover which of the arguments of the next section break down in that case.

As discussed prior to Proposition 1.3 above, one dimensional models with $J_{x-y} \sim 1 /|x-y|^{2}$ have a discontinuity in $P_{\infty}$ at their critical points [AN2]. Because of the result of $[\mathrm{BK}]$ concerning discontinuities (away from the critical point), such models were the most promising candidates, among independent, translation invariant models for nonuniqueness. Proposition 1.1 of this paper shows that even in this situation the infinite cluster is unique.

Proposition 1.1 does not rule out nonuniqueness for directed percolation or for dependent percolation models, for which it remains an open problem. For directed percolation nonuniqueness (or more accurately, nonintersection) could mean, for example, that $C(x)$ and $C(y)$ - the sets of sites reachable by directed paths of occupied directed bonds starting from two sites $x$ and $y$-are both infinite but nevertheless disjoint.

\section{The Connectivity Functions and the Free Energy}

The usual definition of free energy in Statistical Mechanics (see e.g. [Ru]) requires the process of an infinite volume limit. In contrast, the percolation free energy seems to be defined with no labor by the elegant formula (1.3). Our first step in the analysis of the free energy (needed to obtain Proposition 1.4) will seemingly spoil the simplicity of (1.3) by introducing an infinite volume limit process (see also [G1; W]). However, this loss of simplicity will be compensated by a better grasp of the properties of $f$.

For any finite $\Lambda \subset \mathbb{Z}^{d}$ we wish to define clusters according to two different boundary conditions: free and wired. The wired or + -cluster of $x$, denoted $C^{+}(x, \Lambda)$ is the cluster of $x$ obtained when every bond with both endpoints outside of $\Lambda$ is set to be occupied. The free or 0 -cluster of $x$, denoted $C^{0}(x, \Lambda)$ is obtained when every bond with at least one endpoint outside of $\Lambda$ is set to be vacant. Note the following properties of these definitions:

1) For $x$ in $\Lambda, C^{0}(x, \Lambda)$ is always finite, while $C^{+}(x, \Lambda)$ is finite precisely when there is no path of occupied bonds from $x$ to the complement of $\Lambda$.

2) Two sites in $\Lambda$ are in the same 0 -cluster if they are connected within $\Lambda$, 
whereas they are in the same + -cluster if they are either connected in $\Lambda$ or both are connected to $\Lambda^{c}$ (the complement of $\Lambda$ ).

3) For a given site $x$ and bond configuration, $C^{0}(x, \Lambda)$ is a monotone increasing function of $\Lambda$, whereas $C^{+}(x, \Lambda)$ is decreasing in $\Lambda$.

We will henceforth use an asterisk * to denote either 0 of + . We define

$$
P_{n}^{*}(x, \Lambda)=\operatorname{Prob}\left(C^{*}(x, \Lambda) \text { contains exactly } n \text { sites }\right)
$$

and

$$
\begin{aligned}
M^{*}(\Lambda) & =\text { Number of distinct finite } * \text {-clusters totally within } \Lambda \\
& =\text { Number of distinct finite } * \text {-clusters which intersect } \Lambda .
\end{aligned}
$$

Note that $M^{+}(\Lambda)$ is one less than the number of + -clusters which intersect $\Lambda$ whenever there is a path of occupied bonds between $\Lambda$ and $\Lambda^{c}$.

Denoting the number of sites in $\Lambda$ by $|\Lambda|$, we define our finite volume approximants to $f$ as

$$
f_{\Lambda}^{*}=|\Lambda|^{-1} \sum_{x \in \Delta} \sum_{n=1}^{\infty} \frac{1}{n} P_{n}^{*}(x, \Lambda)=|\Lambda|^{-1} \sum_{x \in \Lambda} E\left(\left|C^{*}(x, \Lambda)\right|^{-1}=|\Lambda|^{-1} E\left(M^{*}(\Lambda)\right) .\right.
$$

The following lemma is essentially proven in [G1] and [W].

Lemma 2.1. Let $\Lambda_{k}$ be an increasing sequence of finite rectangles converging to $\mathbb{Z}^{d}$. Then for either choice of boundary condition,

$$
\lim _{k \rightarrow \infty} f_{A_{k}}^{*}=f \equiv \sum_{n=1}^{\infty} \frac{1}{n} P_{n}
$$

Proof. It is clear that $C^{*}\left(x, \Lambda_{k}\right)=C(x)$ whenever $C(x)$, the cluster of $x$, is entirely contained in $\Lambda_{k}$. From this one obtains

$$
\begin{gathered}
\left.|| C^{*}\left(x, \Lambda_{k}\right)\right|^{-1}-\left.|C(x)|^{-1}|\leqq| C^{0}\left(x, \Lambda_{k}\right)\right|^{-1} I(\text { there exists a path of occupied } \\
\text { bonds from } \left.x \text { to the complement of } \Lambda_{k}\right),
\end{gathered}
$$

where $I\left(\right.$ ) denotes the indicator function. The right-hand side decreases to 0 as $\Lambda_{k}$ increases to $\mathbb{Z}^{d}$. (To convince oneself of this when $p_{z} \neq 0$ for infinitely many $z$, it is easiest to separate the cases $C(x)$ finite and $C(x)$ infinite.) Consequently

$$
E\left(\left|C^{*}\left(x, \Lambda_{k}\right)\right|^{-1}\right) \rightarrow E\left(|C(x)|^{-1}\right) \equiv f \quad \text { as } k \rightarrow \infty,
$$

not only for fixed $x$, but uniformly for $x$ 's in $\Lambda_{k}$ whose distance from the complement of $\Lambda_{k}$ tends uniformly to $\infty$. Since the left-hand side of (2.5) is uniformly bounded (by 1), it follows that

$$
f_{\Lambda_{k}}^{*}=\left|\Lambda_{k}\right|^{-1} \sum_{x \in \Lambda_{k}} E\left(\left|C^{*}\left(x, \Lambda_{k}\right)\right|^{-1}\right) \rightarrow f
$$

as desired.

Remark. It is clear from the proof that (2.4) remains valid for any choice of $\Lambda_{k}$ 's converging (not necessarily monotonically) to $\mathbb{Z}^{d}$ in the usual van Hove sense (see e.g. $[\mathrm{Ru}])$-i.e. where for every $D$, the fraction of sites in $\Lambda_{k}$ within a distance $D$ of the 
complement tends to zero. (A similar remark applies to Lemma 2.3 below.)

We shall now turn to the derivatives of the free energy and its approximants. Since $C^{*}(x, \Lambda)$ depends only on those bonds with at least one endpoint in $\Lambda$, it follows by a lattice animal representation of $P_{n}^{*}(x, \Lambda)$ (see Sect. 3 ) that (for the model with $p$ as its single parameter) $f_{A}^{*}$ is a polynomial in $p$ and hence smooth. Then by the relation (1.1) between the $p_{x}$ 's and $\beta$,

$$
\frac{\partial f_{A}^{*}}{\partial \beta}=(1 / 2) \sum_{x \in \mathbb{Z}^{d}} J_{x}\left(1-p_{x}\right) \frac{\partial f_{A}^{*}}{\partial p_{x}},
$$

provided that the use of the chain rule can be justified. (The factor of $1 / 2$ in (2.7) is needed because for each $x \neq 0, p_{x}$ and $p_{-x}$ are not independent parameters, but rather identical.) The validity of the chain rule is part of the next lemma which is the finite volume version of Proposition 1.4. An important role will be played by the connectivity functions

$$
\tau_{\Lambda}^{*}(x, y)=\operatorname{Prob}(x \text { and } y \text { are in the same } * \text {-cluster of } \Lambda) .
$$

We shall write $\{x, y\}$ to denote the bond between $x$ and $y$.

Lemma 2.2. For any finite $\Lambda \subset \mathbb{Z}^{d}$ and either choice of boundary condition $*, f_{\Lambda}^{*}$ is a convex (polynomial) function of $p$ with

$$
\frac{\partial f_{\Lambda}^{*}}{\partial p}=-(1 / 2)|\Lambda|^{-1}(1-p)^{-1} \sum_{z \in \Gamma \times \sum_{\mathbb{Z}}}\left(1-\tau_{\Lambda}^{*}(x, x+z)\right),
$$

where $\sum^{\prime}$ for $*=0$ is restricted to $x$ and $x+z \in \Lambda$ and for $*=+$ to $x$ or $x+z \in \Lambda . f_{\Lambda}^{*}$ is also a convex, differentiable function of $\beta$ with

$$
\frac{\partial f_{\Lambda}^{*}}{\partial \beta}=-(1 / 2)|\Lambda|^{-1} \sum_{x \in \Lambda} \sum_{z \in \mathbb{Z}^{d}} J_{z}\left(1-\tau_{\Lambda}^{*}(x, x+z)\right) .
$$

Proof. For $x$ and $y$ both in $\Lambda$ or for one of $x$ and $y$ in $\Lambda$ and $*=+$, let us denote by $B_{\{x, y\}}^{*}$ the event that $M^{*}(\Lambda)$ depends on the occupation status of the bond $\{x, y\}$. For all other cases, let $B_{\{x, y\}}^{*}$ be the null event. Note that under $B_{\{x, y\}}^{*}, M^{*}(\Lambda)$ decreases by exactly one when $\{x, y\}$ changes from vacant to occupied. Thus by a variant of Russo's formula [Rus], we may differentiate the right-hand side of (2.3) to obtain

$$
\frac{\partial f_{\Lambda}^{*}}{\partial p_{z}}=-|\Lambda|^{-1} \sum_{x \in \mathbb{Z}^{d}} \operatorname{Prob}\left(B_{\{x, x+z\}}^{*}\right) .
$$

Now when $B_{\{x, y\}}^{*}$ is not the null event, it is simply given by

$$
\begin{aligned}
B_{\{x, y\}}^{*}= & \{x \text { and } y \text { are not in the same } * \text {-cluster } \\
& \text { after removal of the bond }\{x, y\}\} .
\end{aligned}
$$

This specific expression for $B_{\{x, y\}}^{*}$ shows that for $x$ in $\Lambda$ and any $z$ in $\mathbb{Z}^{d}$ for $*=+$ or $z$ such that $x+z \in \Lambda$ for $*=0$ :

$$
1-\tau_{\Lambda}^{*}(x, x+z)=\left(1-p_{z}\right) \operatorname{Prob}\left(B_{\{x, x+z\}}^{*}\right) .
$$


Thus (2.10) may be rewritten as

$$
\frac{\partial f_{\Lambda}^{*}}{\partial p_{z}}=-|\Lambda|^{-1}\left(1-p_{z}\right)^{-1} \sum_{x \in \mathbb{Z}^{d}}^{\prime}\left(1-\tau_{\Lambda}^{*}(x, x+z)\right)
$$

which yields (2.8).

The above expression for $B_{\{x, y\}}^{*}$ shows also that $\operatorname{Prob}\left(B_{\{x, x+z\}}^{*}\right)$ is a decreasing function of each of the $p_{y}$ 's, and hence, by (2.10), $f_{A}^{*}$ is convex in each $p_{z}$ and thus (by the chain rule) is convex in $p$. Convexity in $\beta$ follows similarly, once the expression (2.9) (or (2.7)) for $\partial f_{A}^{*} / \partial \beta$ is shown to be valid.

For the case $*=0$, where $f_{\Lambda}^{0}$ depends on only a finite number of bond parameters, (2.9) is an immediate consequence of (2.12) and the chain rule for finitely many variables. For the case $*=+$, where $f_{\Lambda}^{+}$depends on all the bond parameters, we will justify (2.9) when $\sum_{z} J_{z}<\infty$ by showing that $f_{\Lambda}^{+}$as a function of all the $p_{z}$ 's is (Frechet) differentiable in the $L^{1}$ sense; when $\sum_{z} J_{z}=\infty,(2.9)$ is valid since both sides are identically zero.

To see that $f_{\Lambda}^{+}$is $L^{1}$-differentiable, we first note that both $f_{\Lambda}^{+}$and $\tau_{\Lambda}^{+}(x, y)$ are continuous in the $L^{1}$ sense. This is so because these functions fail to be polynomials only through their dependence on the probabilities

$$
\text { Prob \{some bond between } u \text { and } \Lambda^{c} \text { is occupied\}, } u \in \Lambda \text {; }
$$

however these probabilities are clearly $L^{1}$-continuous. The continuity of the connectivity functions $\tau_{\Lambda}^{+}(x, y)$ is even uniform in $x, y$ since as $x$ and $y$ vary over $\mathbb{Z}^{d}$, there are only finitely many distinct functions.

We then use the continuity of $f_{A}^{+}$to write $f_{A}^{+}\left(\left\{p_{y}\right\}\right)-f_{\Lambda}^{+}\left(\left\{\bar{p}_{y}\right\}\right)$ as a telescoping sum in which one variable at a time is incremented. Then by the single variable intermediate value theorem,

$$
f_{\Lambda}^{+}\left(\left\{p_{y}\right\}\right)-f_{\Lambda}^{+}\left(\left\{\bar{p}_{y}\right\}\right)=(1 / 2) \sum_{z \in \mathbb{Z}^{d}}\left(p_{z}-\bar{p}_{z}\right) \frac{\partial f_{\Lambda}^{+}}{\partial p_{z}}\left(\left\{p_{y}^{z}\right\}\right)
$$

where for each $z, p_{z}^{z}$ lies between $p_{z}$ and $\bar{p}_{z}$ while every other $p_{y}^{z}$ is either $p_{y}$ or $\bar{p}_{y}$. It then follows, by (2.10)-(2.11), and the uniform continuity of $\tau_{\Lambda}^{+}$, that as $\left\{p_{y}\right\} \rightarrow\left\{\bar{p}_{y}\right\}$,

$$
f_{\Lambda}^{+}\left(\left\{p_{y}\right\}\right)-f_{\Lambda}^{+}\left(\left\{\bar{p}_{y}\right\}\right)=(1 / 2) \sum_{z}\left(p_{z}-\bar{p}_{z}\right) \frac{\partial f_{\Lambda}^{+}}{\partial p_{z}}\left(\left\{\bar{p}_{y}\right\}\right)+o\left(\sum_{z}\left|p_{z}-\bar{p}_{z}\right|\right) .
$$

This completes the proof.

Although connectivity functions involving more than two sites are not relevant for Proposition 1.4 (whose proof is the main object of this section of the paper), they are treated in the next lemma because of Proposition 1.2. We define

$$
\tau_{\Lambda}^{*}\left(x_{1}, \ldots, x_{n}\right)=\operatorname{Prob}\left(x_{1}, \ldots, x_{n} \text { all belong to the same } * \text {-cluster of } \Lambda\right) \text {; }
$$

analogously, $\tau^{0}$ denotes the ordinary connectivity function $\tau$ while

$$
\begin{aligned}
\tau^{+}\left(x_{1}, \ldots, x_{n}\right)= & \operatorname{Prob}\left(x_{1}, \ldots, x_{n}\right. \text { all belong to the same cluster } \\
& \text { or } \left.C\left(x_{1}\right), \ldots, C\left(x_{n}\right) \text { are all infinite }\right) .
\end{aligned}
$$


Lemma 2.3. With $\Lambda_{k}$ as in Lemma 2.1, either choice of boundary condition and any fixed $n$,

$$
\tau_{\Lambda_{k}}^{*}\left(x_{1}, \ldots, x_{n}\right)-\tau^{*}\left(x_{1}, \ldots, x_{n}\right) \rightarrow 0 \quad \text { as } k \rightarrow \infty
$$

The convergence in (2.14) is uniform for $x_{1}, \ldots, x_{n}$ whose relative distances remain bounded while their distances from the boundary of $\Lambda_{k}$ tend uniformly to $\infty$.

For any fixed $n$ and $x_{1}, \ldots, x_{n}, \tau\left(x_{1}, \ldots, x_{n}\right)$ is a lower semicontinuous function and $\tau^{+}\left(x_{1}, \ldots, x_{n}\right)$ is an upper semicontinuous function of $\left\{p_{z}\right\}$ in the $L^{1}$ sense. Finally, in the one parameter ( $p$ or $\beta$ ) models, $\tau\left(x_{1}, \ldots, x_{n}\right)$ is a left continuous function of $p$ or $\beta$ while $\tau^{+}\left(x_{1}, \ldots, x_{n}\right)$ is right continuous.

Proof. For fixed $x, C^{0}\left(x, \Lambda_{k}\right)$ is increasing in $k$ while $C^{+}\left(x, \Lambda_{k}\right)$ is decreasing in $k$ and $C^{+}\left(x, \Lambda_{k}\right) \supset C^{0}\left(x, \Lambda_{k}\right)$. Further, if $C(x)$ is finite, then $C(x)=C^{*}\left(x, \Lambda_{k}\right)$ for sufficiently large $k$. If $C(x)$ is infinite then $C^{0}\left(x, \Lambda_{k}\right) \rightarrow C(x)$, while $C^{+}\left(x, \Lambda_{k}\right) \rightarrow\{z:|C(z)|=\infty\}$ (cf. proof of Lemma 2.1). It follows that for fixed $x_{1}, \ldots, x_{n}, \tau_{\Lambda_{k}}^{0}\left(x_{1}, \ldots, x_{n}\right)$ increases to $\tau^{0}\left(x_{1}, \ldots, x_{n}\right)$ as $k \rightarrow \infty$, while $\tau_{\Lambda_{k}}^{+}\left(x_{1}, \ldots, x_{n}\right)$ decreases to $\tau^{+}\left(x_{1}, \ldots, x_{n}\right)$ as $k \rightarrow \infty$. The first paragraph of the lemma easily follows.

To obtain the semicontinuity of $\tau^{*}$, we next note that, as discussed in the proof of Lemma 2.2 (for $n=2$ ), for each finite $\Lambda, \tau_{\Lambda}^{*}$ is continuous in the $L^{1}$-sense. But by monotonicity in $\Lambda$,

$$
\tau\left(x_{1}, \ldots, x_{n}\right)=\sup \left\{\tau_{\Lambda}^{0}\left(x_{1}, \ldots, x_{n}\right) \| \Lambda \mid<\infty\right\},
$$

while

$$
\tau^{+}\left(x_{1}, \ldots, x_{n}\right)=\inf \left\{\tau_{\Lambda}^{+}\left(x_{1}, \ldots, x_{n}\right)|| \Lambda \mid<\infty\right\}
$$

Now a function $F\left(\left\{p_{z}\right\}\right)$ is defined to be lower semicontinuous in the $L^{1}$ sense at $\left\{\bar{p}_{z}\right\}$ if

$$
F\left(\left\{\bar{p}_{z}\right\}\right)=\lim _{\delta \rightarrow 0} \inf \left\{F\left(\left\{p_{z}\right\}\right)\left|\sum_{z}\right| p_{z}-\bar{p}_{z} \mid \leqq \delta\right\}
$$

while upper semicontinuity is defined similarly but with inf replaced by sup. It then follows, by standard arguments, from (2.15)-(2.16) and the $L^{1}$-continuity of each $\tau_{\Lambda}^{*}$ that $\tau^{0}$ is lower semicontinuous and $\tau^{+}$is upper semicontinuous in the $L^{1}$ sense (e.g. see [C, Theorem II.8.6]).

Finally, in the one parameter models, $\tau^{*}$ is increasing in $p$ or $\beta$. This makes the lower semicontinuous $\tau^{0}$ left continuous, and the upper semicontinuous $\tau^{+}$right continuous (compare [Rus]).

Proposition 1.4 is now an immediate consequence of the previous three lemmas.

Proof of Proposition 1.4. Let us first consider $f$ as a function of $p$ and view it, by Lemma 2.1, as the limit of $f_{\Lambda}^{0}$ (with e.g. $\Lambda=\Lambda_{k} \rightarrow \mathbb{Z}^{d}$ ). According to Lemmas 2.2 and 2.3, we have the following situation: i) $f$ is a pointwise limit of (smooth) convex functions, ii) the derivatives of those functions converge pointwise, and iii) the limit of the derivatives (which is just the right-hand side of (1.5)) is left continuous. It follows, by general arguments (as in [R], Theorem 25.7), that $f$ is convex with its left derivative given by this limit of derivatives (i.e. (1.5) is valid). The analogous 
argument with 0 replaced by + and left by right yields (1.6). This proves part i) of the theorem.

Part ii) is obtained by essentially the same arguments provided $\sum_{z} J_{z}<\infty$. When $\sum_{z} J_{z}=\infty, f \equiv 0$, and for any $x$ with $J_{x}>0,1-\tau^{+}(0, x)$ is trivially zero while $1-\tau(0, x)$ is zero because the right-hand side of $(1.5)$ is already known to be zero for any choice of $\Gamma$.

Part iii) now follows easily from the identity

$$
\begin{aligned}
\frac{\partial f}{\partial p}(p+0)-\frac{\partial f}{\partial p}(p-0)=[2(1-p)]^{-1} \sum_{x \in \Gamma} \operatorname{Prob}_{0}(0 \text { and } x \text { belong to } \\
\text { distinct infinite clusters }) .
\end{aligned}
$$

\section{Differentiability of the Free Energy}

The purposes of this section are to prove Proposition 1.5 and to present some extensions of it. Here we spoil the simplicity of the expression (1.3) for the free energy, not by introducing infinite volume limits but rather by decomposing $P_{n}$ according to more detailed information about the cluster of the origin, in particular according to the bonds associated with the cluster. Throughout this section, we assume that the $p_{z}$ 's are summable since otherwise $f \equiv 0$ and Proposition 1.5 is vacuously true.

Let us extend the definition of $C(0)$ which was a collection only of sites by defining $\tilde{C}(0)$, the bond cluster of the origin, to be the connected graph whose vertices are the sites of $C(0)$ and whose edges are the occupied bonds incident to any of the sites in $C(0)$. Its boundary, $\partial \widetilde{C}(0)$, is the set of all vacant bonds incident to any site in $C(0)$. The decomposition of $P_{n}$ we will use is based on the number of $\Gamma$-bonds in $\tilde{C}(0)$ and $\partial \widetilde{C}(0)$. By a $\Gamma$-bond we simply mean a pair $\{x, x+z\}$ with $z \in \Gamma$; recall from Sect. 1c that the $\Gamma$-bonds are precisely those bonds whose common occupation probability $p$ is the single varying parameter of the model. We will denote by $|\widetilde{C}|$ the number of sites in $\widetilde{C}$, by $\|\widetilde{C}\|$ the number of $\Gamma$-bonds in $\widetilde{C}$ and by $\|\partial \tilde{C}\|$ the number of $\Gamma$-bonds in $\partial \tilde{C}$. For finite $n, P_{n}$ may then be decomposed as

$$
P_{n}=\sum_{m, l} P_{n m l}
$$

where

$$
P_{n m l}=\operatorname{Prob}(|\tilde{C}(0)|=n,\|\tilde{C}(0)\|=m,\|\partial \tilde{C}(0)\|=l) .
$$

$P_{n m l}$ may be further decomposed according to the possible configurations $\tilde{A}$ (often called lattice animals) of $\widetilde{C}(0)$, using the identity

$$
P(\tilde{C}(0)=\tilde{A})=\prod_{\{x, y\} \in \tilde{A}} p_{y-x} \cdot \prod_{\left\{x^{\prime} y^{\prime}\right\} \in \partial \tilde{A}}\left(1-p_{y^{\prime}-x^{\prime}}\right) .
$$

It follows, by a summation of this identity over those $\tilde{A}$ 's with $|\tilde{A}|=n,\|\tilde{A}\|=m$ and $\|\partial \tilde{A}\|=l$, that

$$
P_{n m l}=a_{n m l} p^{m}(1-p)^{l},
$$

where $a_{n m l} \geqq 0$ is independent of $p$ (but depends on the $p_{z}$ 's with $z$ not in $\Gamma$ ). When $p_{z}=0$ for all $z$ not in $\Gamma$ (as in nearest neighbor bond percolation), $a_{n m l}$ is just 
the number of lattice animals with the specified $n, m$ and $l$. Note that, in any case,

$$
a_{n m l}=0 \quad \text { unless } m+l \leqq n|\Gamma|
$$

(and unless $m+l \geqq n|\Gamma| / 2$ ).

We may now differentiate the free energy (1.3) by using the lattice animal representation, (3.1) and (3.4), to obtain the formal identity,

$$
\frac{\partial f}{\partial p}=\sum_{n, m, l} n^{-1}[m / p-l /(1-p)] a_{n m l} p^{m}(1-p)^{l},
$$

or equivalently,

$$
\left.\frac{\partial f}{\partial p}=E\left(|\tilde{C}(0)|^{-1}[\|\tilde{C}(0)\| / p-\|\partial \tilde{C}(0)\| /(1-p)]\right) I(|\tilde{C}(0)|<\infty)\right),
$$

where $I(B)$ denotes the indicator function of the event $B$. The remainder of this section is devoted to showing that the right-hand side of (3.6) is sufficiently convergent to conclude that $f$ is continuously differentiable in $p$.

Our analysis is based on an estimation of the smallness of the "surface-volume difference" term, $[m / p-l /(1-p)]$, of (3.6) or the analogous term of (3.7). This smallness is related to previous results on the surface to volume ratio being $(1-p) / p$ for large finite clusters [KSo] or for infinite clusters (e.g. [G2; NS1]). (See also $[\mathrm{L} 1 ; \mathrm{L} 2 ; \mathrm{Ha}]$.$) As mentioned in a remark of the introduction, the essential$ ingredients of our analysis are already contained in the proof given in [K1, p. 252] of twice-differentiability of $f$ for standard two-dimensional models. Indeed, the estimates on $[m / p-l /(1-p)]$ lead not only to differentiability of $f$ (Proposition 1.5) but also to sufficient conditions for twice-differentiability and related critical exponent inequalities (see Proposition 3.3 and its corollaries below). These and related estimates can also be used to obtain other critical exponent equalities (see e.g. $[\mathrm{N} 1 ; \mathrm{N} 2])$.

The basic estimate needed is essentially a large deviation estimate on the random variable $[\|\tilde{C}(0)\| / p-\|\partial \tilde{C}\| /(1-p)]$. There are at least two approaches to obtaining this estimate. One is a purely combinatorial approach based on (3.4), as in [K1, Lemma 5.1]; the other uses an algorithmic construction of percolation clusters which was applied in [KSh] to the surface to volume ratio of infinite clusters. Both approaches reduce the estimate to a standard large deviation result for binomial random variables. Here we emphasize the second approach. We write $\operatorname{Prob}_{p^{\prime}}, E_{p^{\prime}}$, etc. when we want to refer to a particular value $p^{\prime}$ for the $\Gamma$-bond occupation probability.

Lemma 3.1. For any $\alpha \geqq 0$,

$$
\begin{aligned}
\operatorname{Prob}_{p}(|[\|\tilde{C}(0)\| / p-\|\partial \tilde{C}(0)\| /(1-p)]| & \geqq \alpha k,\|\tilde{C}(0)\|+\|\partial \tilde{C}(0)\|=k) \\
& \leqq 2 \exp \left(-k H(p) \alpha^{2} / 2\right),
\end{aligned}
$$

where $H(p)$ is uniformly positive as a function of $p$ on compact subsets of $(0,1)$.

Proof. This estimate is based on the following relations. For any $0<p<1$ and any real $r$, we have the identity

$$
\begin{aligned}
& E_{p}(\exp \{r[\|\tilde{C}(0)\| / p-\|\partial \tilde{C}(0)\| /(1-p)]\} I(\|\tilde{C}(0)\|+\|\partial \tilde{C}(0)\|=k)) \\
& \quad=\left[p e^{r / p}+(1-p) e^{-r /(1-p)}\right]^{k} \operatorname{Prob}_{p^{\prime}}(\|\tilde{C}(0)\|+\|\partial \tilde{C}(0)\|=k)
\end{aligned}
$$


where

Furthermore,

$$
p^{\prime}=p e^{r / p}\left[p e^{r / p}+(1-p) e^{-r /(1-p)}\right]^{-1}
$$

$$
\begin{gathered}
\operatorname{Prob}_{p}(\|\tilde{C}(0)\|=m,\|\partial \tilde{C}(0)\|=l)=\sum_{n} P_{n m l} \\
\quad=\left(\sum_{n} a_{n m l}\right) p^{m}(1-p)^{l} \leqq\left(\begin{array}{c}
m+l \\
m
\end{array}\right) p^{m}(1-p)^{l} .
\end{gathered}
$$
Then

To prove the relations (3.9) and (3.11), write $P_{m l}(p)$ for the left-hand side of (3.11).

$$
\begin{aligned}
\operatorname{LHS} \text { of }(3.9) & =\sum_{\substack{m, l \\
m+l=k}}\left(e^{r / p}\right)^{m}\left(e^{-r /(1-p)}\right)^{l} P_{m l}(p) \\
& =\left[p e^{r / p}+(1-p) e^{-r /(1-p)}\right]^{k} \sum_{\substack{m, l \\
m+l=k}}\left(p^{\prime} / p\right)^{m}\left(\left(1-p^{\prime}\right) /(1-p)\right)^{l} P_{m l}(p),
\end{aligned}
$$

where $p^{\prime}$ is given by (3.10). But (3.4) implies that for any $p^{\prime}$ in $(0,1)$,

$$
P_{m l}\left(p^{\prime}\right)=\left(p^{\prime} / p\right)^{m}\left(\left(1-p^{\prime}\right) /(1-p)\right)^{l} P_{m l}(p)
$$

so that (3.12) yields (3.9).

Our derivation of (3.11) is based on the following construction. (Similar constructions of clusters have been used previously, as in [PS; KSh] and variants appear at least as far back as [H2].) Choose some total ordering of all $\Gamma$-bonds, and let $W_{1}, W_{2}, \ldots$ be a sequence of independent 0 or 1 valued random variables with $P\left(W_{i}=1\right)=p$ for each $i$. A growing sequence of partial clusters, $\tilde{C}_{1}, \tilde{C}_{2}, \ldots$ which converge to $\widetilde{C}(0)$ (if it is finite) is constructed along with their partial boundaries, $\partial \widetilde{C}_{j}$, as follows. $\tilde{C}_{1}$ is the graph consisting of the site 0 and no bonds, and $\partial \tilde{C}_{1}$ is empty. $\widetilde{C}_{n+1}$ and $\partial \widetilde{C}_{n+1}$ are constructed from $\tilde{C}_{n}$ and $\partial \tilde{C}_{n}$ in several steps. First, occupation variables are assigned to those $\Gamma$-bonds not in $\widetilde{C}_{n} \cup \partial \widetilde{C}_{n}$ but which are incident to some site in $\widetilde{C}_{n}$ by assigning the next available "unused" $W_{i}$-values to the $\Gamma$-bonds according to the chosen order; e.g. $W_{1}$ is assigned to the $\Gamma$-bond of lowest order among those incident to the origin. Second, occupation values are assigned to non$\Gamma$-bonds not in $\widetilde{C}_{n} \cup \partial \widetilde{C}_{n}$ which are incident to some site in $\widetilde{C}_{n}$. Of course $\{x, x+z\}$ is assigned to be occupied at this stage with probability $p_{z}$. Third $\widetilde{C}_{n}$ is augmented by the "newly occupied" $\Gamma$ and non- $\Gamma$-bonds and $\partial \widetilde{C}_{n}$ is similarly augmented by the "newly vacant" bonds.

Now, the event that $\|\widetilde{C}(0)\|=m$ and $\|\partial \tilde{C}(0)\|=l$, is identical to the event that the above process terminates with exactly $m+l W_{i}$-values "used," exactly $m$ of which are equal to 1 . Thus

$$
P_{m l}(p) \leqq \operatorname{Prob}_{p}\left(\sum_{i=1}^{m+l} W_{i}=m\right)=\left(\begin{array}{c}
m+l \\
m
\end{array}\right) p^{m}(1-p)^{l},
$$

which gives (3.11).

To obtain (3.8) we first note that either (3.9) or (3.11) implies that

$$
\text { LHS of }(3.9) \leqq\left[p e^{r / p}+(1-p) e^{-r /(1-p)}\right]^{k} \text {. }
$$


The remainder of the argument, which we discuss briefly, is quite standard. Let us define $H(p)$ as the largest $H$ such that

$$
p e^{r / p}+(1-p) e^{-r /(1-p)} \leqq \exp \left[r^{2} /(2 H(p))\right] \text { for all real } r \text {. }
$$

$H(p)>0$ because the left-hand side of $(3.16)=1+0\left(r^{2}\right)$ for small $r$. By Chebyshev's inequality and (3.15)-(3.16), for any $t \geqq 0$,

$$
\begin{aligned}
\text { LHS of }(3.8) & \leqq e^{-t \alpha k}\left[\left.(\operatorname{LHS} \text { of }(3.9))\right|_{r=t}+\left.(\operatorname{LHS} \text { of }(3.9))\right|_{r=-t}\right] \\
& \leqq 2 \exp \left[k t^{2} /(2 H(p))-k \alpha t\right] .
\end{aligned}
$$

Minimizing this last expression over $t$ yields (3.8).

Remarks. i) The basic estimate (3.8) involves the rather implicitly defined $H(p)$. A more explicit estimate is given in [K1, Lemma 5.1]. One can both improve (3.8) and make it more explicit by avoiding the wasteful estimate (3.16) for the binomial generating function. This would replace $H(p) \alpha^{2} / 2$ in (3.8) by an explicit binomial entropy function.

ii) In the case of ordinary bond percolation on a graph with all bonds (of the graph) having a common occupation probability $p$, the inequality (3.11) implies that $a_{m l}$, the number of (bond) lattice animals with volume $m$ and surface $l$ is bounded by $\left(\begin{array}{c}m+l \\ m\end{array}\right)$. Thus (3.11) is a generalization of (and a very slight improvement over) the usual result, $a_{m l} \leqq(m+l)^{m+l} m^{-m} l^{-l}$ (which follows from $P_{m l}(p) \leqq 1$ for all $p$ ).

Lemma 3.2. Given any $0<p_{1}<p_{2}<1$, and any constant $K_{1}<\infty$, there exist finite constants $K_{2}, K_{3}$ so that for all $p$ in $\left[p_{1}, p_{2}\right]$ and all $n \geqq 1$,

$$
\operatorname{Prob}_{p}\left(|\tilde{C}(0)|=n,|[\|\tilde{C}(0)\| / p-\|\partial \tilde{C}(0)\| /(1-p)]| \geqq K_{2}(n \ln (n))^{1 / 2}\right) \leqq K_{3} n^{-K_{1}} .
$$

Proof. By (3.5) and (3.8),

$$
\begin{aligned}
\text { LHS of }(3.18) & \leqq \sum_{k=1}^{n|\Gamma|} 2 \exp \left(-k H(p)\left[K_{2}(n \ln (n))^{1 / 2} / k\right]^{2} / 2\right) \\
& \leqq 2 n|\Gamma| \exp \left(-\left(K_{2}\right)^{2}(2|\Gamma|)^{-1} H(p) \ln (n)\right) \\
& \leqq 2|\Gamma| n^{-\left[\left(K_{2}\right)^{2} H(p) /(2|\Gamma|)\right]+1}
\end{aligned}
$$

With an appropriate choice of $K_{2}$, this immediately yields $(3.18)$ since $H(p)$ is uniformly positive on $\left[p_{1}, p_{2}\right]$.

Proof of Proposition 1.5. Since, by (3.4)-(3.5), $P_{n}(p)$ is a polynomial in $p$, and hence continuously differentiable, it suffices (by standard arguments) to show that for any $0<p_{1}<p_{2}<1$

$$
\sum_{n=1}^{\infty} n^{-1} \partial P_{n} / \partial p \text { is uniformly convergent on }\left[p_{1}, p_{2}\right]
$$

in order to conclude that $f$ is continuously differentiable with $\partial f / \partial p=\sum_{n} n^{-1} \partial P_{n} / \partial p$, and thus prove Proposition 1.5. 
Now by (3.4),

$$
\partial P_{n} / \partial p=\sum_{m, l}[m / p-l /(1-p)] a_{n m l} p^{m}(1-p)^{l} .
$$

We break up this sum into two parts, depending on whether $|m / p-l /(1-p)|<$ $K_{2}(n \ln (n))^{1 / 2}$ or not. In the part where this inequality is violated, we use the bound (see (3.5))

$$
\begin{aligned}
&|m / p-l /(1-p)| \leqq(m+l) /[p(1-p)] \leqq|\Gamma| n /[p(1-p)] . \text { This yields } \\
&\left|\partial P_{n} / \partial p\right| \leqq K_{2}(n \ln (n))^{1 / 2} P_{n}+(|\Gamma| n /[p(1-p)]) \cdot(\text { LHS of }(3.18)),
\end{aligned}
$$

so that by (3.18),

$$
\left|\sum_{n=n_{1}}^{n_{2}} n^{-1} \partial P_{n} / \partial p\right| \leqq O\left(\left[\ln \left(n_{1}\right) / n_{1}\right]^{1 / 2}\right) \cdot \sum_{n \geqq n_{1}} P_{n}+O\left(\left(n_{1}\right)^{-K_{1}+1}\right)
$$

for all $p$ in $\left[p_{1}, p_{2}\right]$. By choosing $K_{2}$ so that $K_{1}>1$, this yields (3.20).

For the remainder of this section of the paper, we apply the methods used in the proof of Proposition 1.5 to obtain bounds on the second and higher derivatives of $f$. The bounds will yield sufficient conditions for $f$ to be twice differentiable (as is known in two-dimensional models [K1, p. 252]) as well as inequalities relating the critical exponents $\alpha, \beta$ and $\delta$. These exponents may be heuristically defined by

$$
\begin{gathered}
f^{\prime \prime \prime}(p) \sim\left(p_{c}-p\right)^{-(1+\alpha)} \quad \text { as } p \uparrow p_{c}, \\
\chi(p) \equiv \sum_{n=1}^{\infty} n P_{n}(p) \sim\left(p_{c}-p\right)^{-\gamma} \quad \text { as } p \uparrow p_{c}, \\
P_{n}\left(p_{c}\right) \sim n^{-(1+1 / \delta)} \quad \text { as } n \rightarrow \infty .
\end{gathered}
$$

The inequalities on the critical exponents, which are valid under various assumptions given in the corollaries to Proposition 3.3 below, are:

$$
\begin{gathered}
\gamma<\infty \quad \text { implies } \alpha \leqq 0, \\
P_{\infty}\left(p_{c}\right)=0 \quad \text { and } \delta<\infty \quad \text { imply } \alpha \leqq 0, \\
P_{\infty}\left(p_{c}\right)=0 \quad \text { implies } 1+\alpha \leqq\left(\frac{\delta-2}{\delta-1}\right) \frac{\gamma}{2} .
\end{gathered}
$$

Note that the right-hand side of (3.27) is non-negative since $\delta \geqq 2$ [AB1]. We remark that analogues of these inequalities are valid with $\alpha$ and $\gamma$ replaced by $\alpha^{\prime}$ and $\gamma^{\prime}$, the analogous exponents defined when $p \downarrow p_{c}$ (but some strengthening of the hypotheses of (3.26) - (3.27) is needed).

The free energy $f(p)$ is infinitely differentiable, in fact real analytic, for $p<p_{c}$. This follows from the lattice animal representation ((3.1)-(3.5)) and the known facts that $\chi(p)<\infty$ for $p<p_{c}$ [AB1] and that $\chi(p)<\infty$ implies exponential decay of $P_{n}(p)$ [H1; K1, Theorem 5.1; AN1]. At $p=p_{c}, f$ is not expected to be more than twice differentiable with $f^{\prime \prime \prime}$ diverging according to some critical exponent $\alpha$, as in (3.22). The following technical proposition gives bounds on the $k$ th derivative, $f^{(k)}(p)$, in 
terms of moments of the finite cluster size distribution. We define for real $r$ and $t$

$$
\chi(p ; r, t)=\sum_{1 \leqq n<\infty} n^{r}[\ln (n)]^{t} P_{n}(p) .
$$

Note that $\chi(p ; 1,0)$ is just the expected size of finite clusters.

Proposition 3.3. In a translation invariant model, suppose that for some $0<p_{1}<p_{2}<\infty$, some $\sigma>0$, and some $k=2,3,4, \ldots$

$$
\sup _{p_{1}<p<p_{2}} \chi(p ;(k / 2)-1,(k / 2)+\sigma)<\infty .
$$

Then $f(p)$ is $k$-times continuously differentiable on $\left(p_{1}, p_{2}\right)$ and for some $B<\infty$, depending on $p_{1}, p_{2}$ and $k$ only,

$$
\left|f^{(k)}(p)\right| \leqq B[\chi(p ;(k / 2)-1, k / 2)+1] \text { for } p \text { in }\left(p_{1}, p_{2}\right) .
$$

Proof. Extending the lattice animal expansion (3.21) for $\partial P_{n} / \partial p$ to higher derivatives yields inductively that $\partial^{k} P_{n} / \partial p^{k}$ is a finite linear combination (whose coefficients are independent of $n$ ) of terms of the form

$$
F_{i j k^{\prime}}(p) \sum_{m, l} m^{i} l^{j}[m / p-l /(1-p)]^{k^{\prime}} P_{n m l}(p),
$$

where

$$
k^{\prime}+2(i+j) \leqq k
$$

and $F_{i j k^{\prime}}$ is a polynomial in $p^{-1}$ and $(1-p)^{-1}$. The conclusions of the proposition now follow by essentially the same arguments as used in the proof of Proposition 1.5.

As corollaries of Proposition 3.3, we now give in order precise versions of the critical exponent inequalities (3.25)-(3.27).

Corollary 3.4. In a translation invariant model with $p_{c}>0$,

$$
f^{\prime \prime}(p)=O(\ln (\chi(p))) \quad \text { as } p \uparrow p_{c} .
$$

Proof. By the $k=2$ case of Proposition 3.3, we have

$$
f^{\prime \prime}(p)=O\left(\sum_{n=1}^{\infty} \ln (n) P_{n}(p)\right)
$$

which yields the desired result by the concavity of $\ln (u)$ and Jensen's inequality.

Remark. When $\gamma<\infty$, (3.31) shows that $f^{\prime \prime}(p)$ cannot have a power law divergence as $p \uparrow p_{c}$ and hence that $\alpha \leqq 0$. If $\gamma=\infty$ (which may be the case in one dimensional $1 /|x-y|^{2}$ models), (3.31) still gives useful information; e.g., if

$$
\chi(p)=\exp \left[O\left(\left(p_{c}-p\right)^{-\theta}\right)\right] \quad \text { as } p \uparrow p_{c},
$$

then $\alpha \leqq \theta$. Conversely if one could show that $f^{\prime \prime}$ had a power law divergence, that would prove exponential divergence of $\chi$.

Corollary 3.5. In a translation invariant model with $p_{c}>0$ and $P_{\infty}\left(p_{c}\right)=0$, if

$$
\sum_{n=1}^{\infty} \ln (n) P_{n}\left(p_{c}\right)<\infty
$$


then

$$
f^{\prime \prime}(p)=O(1) \quad \text { as } p \uparrow p_{c} .
$$

Proof. Let $b_{n}=\ln (n)-\ln (n-1)>0$. Since

$$
P_{\geqq n}(p) \equiv \sum_{m \geqq n} P_{m}(p)
$$

is increasing in $p$, it follows, by the hypotheses of this corollary, that for $p \leqq p$,

$$
\begin{aligned}
\sum_{n=1}^{\infty} \ln (n) P_{n}(p) & =\sum_{n>1} b_{n} P_{\geqq n}(p) \leqq \sum_{n>1} b_{n} P_{\geqq n}\left(p_{c}\right) \\
& =\sum_{n=1}^{\infty} \ln (n) P_{n}\left(p_{c}\right)<\infty .
\end{aligned}
$$

Thus, by the $k=2$ case of Proposition 3.3 (i.e. by (3.32)), $f^{\prime \prime}(p)$ is $O(1)$ as $p \uparrow p_{c}$.

Corollary 3.6. In a translation invariant model with $p_{c}>0$ and $P_{\infty}\left(p_{c}\right)=0$, suppose there is some $\delta$ in $[2, \infty)$ so that

Then for any $\varepsilon^{\prime}>0$,

$$
\sum_{n=1}^{\infty} n^{1 / \delta} P_{n}\left(p_{c}\right)<\infty
$$

$$
f^{(3)}(p)=O\left([\chi(p)]^{(\delta-2) /(2 \delta-2)+\varepsilon^{\prime}}\right) \quad \text { as } p \uparrow p_{c} .
$$

Proof. By the $k=3$ case of Proposition 3.3, we have

$$
f^{(3)}(p)=O(\chi(p ; 1 / 2,3 / 2))=O\left(\chi\left(p ; r^{\prime}, 0\right)\right)
$$

for any $r^{\prime}>1 / 2$. By Hölder's inequality, $\chi(p ; r, 0)$ is a log-convex function of $r$, so that for $r^{\prime}=(1-a)(1 / \delta)+a \cdot 1$ with

$$
a=\frac{\delta-2}{2 \delta-2}+\varepsilon^{\prime}
$$

we have

$$
\chi\left(p ; r^{\prime}, 0\right) \leqq(\chi(p ; 1 / \delta, 0))^{1-a} \cdot(\chi(p))^{a} .
$$

But by the assumptions of this corollary and arguments as in the proof of Corollary $3.5, \chi(p ; 1 / \delta, 0)$ is $O(1)$ as $p \uparrow p$, so that (3.36) follows.

Remark. Without assuming either that $P_{\infty}\left(p_{c}\right)=0$ or that (3.35) is valid for some $\delta<\infty$, one may use the $\delta=\infty$ version of the proof of Corollary 3.6 to conclude that

$$
f^{(3)}(p)=O\left([\chi(p)]^{(1 / 2)+\varepsilon^{\prime}}\right) \quad \text { as } p \uparrow p_{c},
$$

so that

$$
1+\alpha \leqq \gamma / 2
$$

This is an improvement of (3.25) (i.e. $\gamma<\infty$ implies $\alpha \leqq 0$ ) only when $\gamma<2$ (which cannot be valid unless $\left.P_{\infty}\left(p_{c}\right)=0[\mathrm{~N} 1 ; \mathrm{N} 2]\right)$. Another exponent inequality,

$$
2+\alpha \leqq \gamma,
$$


can also be derived without assuming $P_{\infty}\left(p_{c}\right)=0$ but again this does not improve the $\alpha \leqq 0$ result unless $\gamma<2$. The precise version of (3.39) is

$$
f^{(4)}(p)=0\left(\chi(p) \cdot\left(p_{c}-p\right)^{-\varepsilon^{\prime}}\right) \quad \text { as } p \uparrow p_{c} .
$$

Its proof, which requires the assumption that for some $r>1$ and $\gamma_{r}<\infty$,

$$
\sum_{1 \leqq n<\infty} n^{r} P_{n}(p)=O\left(\left(p_{c}-p\right)^{-\gamma_{r}}\right) \quad \text { as } p \uparrow p_{c},
$$

is based on the $k=4$ case of Proposition 3.3 and the log convexity of $\chi\left(p ; r^{\prime}, 0\right)$ as a function of $r^{\prime}$.

\section{Derivation of Propositions 1.1, 1.2 and 1.3}

In this section we explain the few extra ingredients needed to obtain Propositions 1.1-1.3 from the results of the last two sections (specifically from Proposition 1.4, Proposition 1.5 and Lemma 2.3).

Proof of Proposition 1.1. We assume here that $\sum_{z} p_{z}<\infty$; see Remark iv) following Proposition 1.5 for the proof when this condition fails. Proposition 1.1 concerns a model with given $p_{z}$ 's and no specified $\Gamma$. We may pick any $z \neq 0$ in $\mathbb{Z}^{d}$ with $p_{z} \neq 0$ and choose $\Gamma=\{z,-z\}$. Then by Propositions 1.4 and 1.5 (with $p=p_{z}$ ), we see that for any $z, p_{z} \neq 0$ implies

$$
\operatorname{Prob}(0 \text { and } z \text { belong to distinct infinite clusters })=0 \text {. }
$$

(If $p=p_{z}=1$, we cannot use Propositions 1.4 and 1.5, but then (4.1) is trivial.) Let us denote by $G(0, z)$ the event whose probability appears in (4.1). If we can show that $S \equiv\{z: \operatorname{Prob}(G(0, z))=0\}$ is closed under addition, then by the irreducibility of the model, $S=\mathbb{Z}^{d}$ and the proposition is proved. So assume that $z$ and $z^{\prime}$ are in $S$ but $z^{\prime \prime}=z+z^{\prime}$ is not in $S$. We wish to derive a contradiction.

Let $x_{0}, x_{1}, x_{2}, \ldots, x_{k}$ be a sequence of distinct sites leading from $z\left(=x_{0}\right)$ to $0\left(=x_{k}\right)$ such that $p_{x_{j}-x_{j-1}}>0$ for each $j$ (such a sequence exists by irreducibility). $\operatorname{Prob}\left(G\left(0, z^{\prime \prime}\right)\right)>0$ by assumption. For each configuration $\omega$ in $G\left(0, z^{\prime \prime}\right)$, define $K=K(\omega)$ as the smallest $j$ such that $x_{j}$ is in $C(0) \cup C\left(z^{\prime \prime}\right)$; since $C(0)$ and $C\left(z^{\prime \prime}\right)$ are disjoint, $x_{K}$ is in only one of these clusters. Define the mapping $\Phi$ on configurations in $G\left(0, z^{\prime \prime}\right)$ by the rule that $\Phi$ forces the bond $\left\{x_{j-1}, x_{j}\right\}$ to be occupied for each $j \leqq K$ (if it was already occupied, $\Phi$ leaves it so) and $\Phi$ leaves unchanged all other bonds.

$\Phi$ has two important properties. First, $\Phi\left(G\left(0, z^{\prime \prime}\right)\right) \equiv\left\{\Phi(\omega): \omega \in G\left(0, z^{\prime \prime}\right)\right\}$ is a subset of the event $G(0, z) \cup G\left(z, z^{\prime \prime}\right)$. Second, since $\Phi$ involves only those bonds from a finite nonrandom collection (i.e. $\left\{\left\{x_{j-1}, x_{j}\right\}: j \leqq k\right\}$ ), all of which have positive occupation probability, it follows that $\operatorname{Prob}(\Phi(G))>0$ for any event $G$ of nonzero probability (see, e.g., [NS1, Proposition 9] for a proof of this claim). These two properties together imply that at least one of the following two probabilities is nonzero: $\operatorname{Prob}(G(0, z))$ and $\operatorname{Prob}\left(G\left(z, z^{\prime \prime}\right)\right)=\operatorname{Prob}\left(G\left(0, z^{\prime}\right)\right)$. But this contradicts the assumption that $z$ and $z^{\prime}$ are in $S$.

Proof of Proposition 1.2. Recalling the definition (2.13) of $\tau^{+}\left(x_{1}, \ldots, x_{n}\right)$, we see that 
the uniqueness of infinite clusters (i.e. the already proved Proposition 1.1) implies

$$
\tau\left(x_{1}, \ldots, x_{n}\right)=\tau^{+}\left(x_{1}, \ldots, x_{n}\right) \text {. }
$$

But by Lemma 2.3, $\tau$ is lower semicontinuous and $\tau^{+}$is upper semicontinuous in the $L^{1}$ sense. Thus $\tau=\tau^{+}$is $L^{1}$-continuous. This yields all of Proposition 1.2 except for the continuity in $\beta$ when $\sum_{z} J_{z}=\infty$. When $\sum_{z} J_{z}=\infty, \tau=\tau^{+}=1$ for all $\beta>0$.

We complete this section with a proof of Proposition 1.3, the continuity of $P_{\infty}$ above threshold. This is a corollary of Proposition 1.1 and the result of van den Berg and Keane [BK] that uniqueness strictly above threshold implies continuity of $P_{\infty}$. Since the context of [BK] was continuity as a function of $p$, we give, for the sake of completeness, a version of the argument of van den Berg and Keane for the proof of continuity of $P_{\infty}$ as a function of $\beta$.

Proof of Proposition 1.3. We first note that since $P_{\infty}$ is a decreasing limit, as $\Lambda_{k} \uparrow \mathbb{Z}^{d}$, of the continuous increasing functions $\operatorname{Prob}\left(C(0) \cap \Lambda_{k}^{c} \neq \varnothing\right) \equiv \operatorname{Prob}$ $\left(\left|C^{+}\left(0, \Lambda_{k}\right)\right|=\infty\right), P_{\infty}$ is necessarily right continuous. To relate left continuity in $\beta$ to uniqueness, we proceed, in a variant of the approach of $[\mathrm{BK}]$, by embedding the one parameter family of models into a single system consisting of independent random variables, $W_{\{x, y\}}$, with exponential distributions. When $J_{y-x}>0$, the distribution is given by

$$
\operatorname{Prob}\left(W_{\{x, y\}} \leqq w\right)=1-\exp \left(-J_{y-x} w\right), \quad \text { for } w \geqq 0 ;
$$

when $J_{y-x}=0$, then $W_{\{x, y\}} \equiv \infty$. (Such an embedding was proposed, in the context of invasion percolation, in [CCN, Sect. 5]).

For any $\beta$ in $(0, \infty)$, we obtain the original parameter- $\beta$ percolation model by considering $\{x, y\}$ to be occupied if $W_{\{x, y\}} \leqq \beta$. We will call a cluster $\tilde{C}$ from this model a $\beta$-cluster with the understanding that each occupied bond still is associated with its $W$-value. We will call an infinite $\beta$-cluster fragile if for every $\beta^{\prime}<\beta$, the removal from the cluster of all the bonds with $W$-values in $\left(\beta^{\prime}, \beta\right]$ leaves only finite components. Analogously to $[\mathrm{BK}]$, we claim that

$$
\begin{aligned}
P_{\infty}(\beta)-P_{\infty}(\beta-0)= & \text { Prob (the origin belongs to } \\
& \text { a fragile infinite } \beta \text {-cluster). }
\end{aligned}
$$

Once this is proved, left continuity follows for $\beta>\beta_{c}$ because by Proposition 1.1 the $\beta$-cluster is unique, and hence cannot be fragile for $\beta>\beta_{c}$ since there is an infinite $(\beta$ $-\varepsilon$ )-cluster for small $\varepsilon$, which must be a subcluster of the $\beta$-cluster.

Since

$$
P_{\infty}\left(\beta^{\prime}\right)=\operatorname{Prob}\left(\text { the } \beta^{\prime}\right. \text {-cluster of the origin is infinite), }
$$

it follows that

$$
\begin{aligned}
P_{\infty}(\beta)-P_{\infty}(\beta-0)= & \operatorname{Prob}(\text { the } \beta \text {-cluster of the origin is } \\
& \text { infinite but the } \beta^{\prime} \text {-cluster of the } \\
& \text { origin is finite for every } \left.\beta^{\prime}<\beta\right) .
\end{aligned}
$$

This shows that LHS of (4.3) $\geqq$ RHS of (4.3). To obtain the opposite inequality, it suffices to show that conditioned on the event that the $\beta$-cluster of the origin is 
infinite and nonfragile, then the conditional probability that there is some infinite $\beta^{\prime \prime}$ path starting at the origin tends to one as $\beta^{\prime \prime} \uparrow \beta$. This follows by standard arguments, since for $\varepsilon$ sufficiently small there is with probability one an infinite $(\beta-\varepsilon)$-cluster inside the infinite $\beta$-cluster of the origin and the conditional probability that there is a $\beta$-path, within the finite region $\Lambda$, from the origin to this infinite $(\beta-\varepsilon)$-cluster tends to one as $\Lambda \uparrow \mathbb{Z}^{d}$.

\section{Site Percolation}

In this section we show that all our results, with suitable adjustments, extend to ordinary nearest neighbor site percolation on $\mathbb{Z}^{d}$. The same arguments apply to site percolation on other $d$-dimensional lattices. At the end of this section, we briefly discuss mixed site-bond percolation models.

The $d$-dimensional site model may be defined by independent variables $\left\{n_{x}: x \in \mathbb{Z}^{d}\right\}$ taking the values 1 (site occupied) or 0 (site vacant) with $\operatorname{Prob}\left(n_{x}=1\right)=p$ for all $x . C(x)$ denotes the set of occupied sites $y$ which are connected to $x$ by nearest neighbor paths over occupied sites; if $x$ is vacant, then $C(x)$ is empty. As usual, one defines

$$
P_{n}=P_{n}(p)=\operatorname{Prob}(|C(0)|=n) \text { for } n=0,1,2, \ldots, \infty
$$

and

$$
\begin{array}{r}
\tau\left(x_{1}, \ldots, x_{n}\right)=\operatorname{Prob}\left(x_{1}, \ldots, x_{n}\right. \text { are all occupied and all } \\
\text { belong to the same cluster }) .
\end{array}
$$

The free energy is defined as

$$
f=f(p)=\sum_{n \geqq 1} n^{-1} P_{n}=E\left(|C(0)|^{-1} I(|C(0)|>0)\right)
$$

and represents the expected number of (nonempty) clusters per site. For a finite $\Lambda \subset \mathbb{Z}^{d}$, we define $C^{0}(x, \Lambda)$ as the $C(x)$ obtained when all sites outside of $\Lambda$ are set to be vacant. We define $C^{+}(x, \Lambda)$ to be the ordinary $C(x)$ if $x \in \Lambda$ and $C(x) \subset \Lambda$; otherwise it is defined as $\Lambda^{c} \cup\left\{y \in \Lambda: C(y) \cap \Lambda^{c} \neq \varnothing\right\}$. If $\Lambda$ is such (e.g. a cube) that $\Lambda^{c}$ (with all its nearest neighbor bonds) has only a single connected component, then $C^{+}(x, \Lambda)$ may be equivalently defined as the cluster of $x$ when all sites in $\Lambda^{c}$ are set to be occupied. Note that $C^{+}(x, \Lambda) \supset C^{0}(x, \Lambda)$ and $C^{*}(x, \Lambda)$ is increasing in $\Lambda$ for $*=0$ and decreasing in $\Lambda$ for $*=+. M^{*}(\Lambda)$ denotes the number of nonempty finite $*$-clusters. $f_{A}^{*}$ is defined as

$$
f_{\Lambda}^{*}=|\Lambda|^{-1} \sum_{z \in \Lambda} E\left(\left|C^{*}(x, \Lambda)\right|^{-1} I\left(\left|C^{*}(x, \Lambda)\right|>0\right)\right)=|\Lambda|^{-1} E\left(M^{*}(\Lambda)\right) .
$$

The analogue of Lemma 2.1 remains valid (with the same proof) in the site percolation context. What changes is the formula for $\partial f_{\Lambda}^{*} / \partial p$. Let us define for $x \in \Lambda$, $N^{*}(x, \Lambda)$ as the number of distinct (finite or infinite) $*$-clusters, obtained after setting $x$ to be vacant, which contain a nearest neighbor to $x$. Note that if $N^{*}(x, \Lambda)=0$, then changing $x$ from vacant to occupied increases $M^{*}(\Lambda)$ by 1 , but if $N^{*}(x, \Lambda) \geqq 1$, then such a change in $x$ decreases $M^{*}(\Lambda)$ by $N^{*}(x, \Lambda)-1$. It follows (see also [G2, 
Theorem 4.3]) that

$$
\begin{aligned}
\partial f_{\Lambda}^{*} / \partial p & =|\Lambda|^{-1} \sum_{x \in \Lambda} E\left(I\left(N^{*}(x, \Lambda)=0\right)+\left(1-N^{*}(x, \Lambda)\right) I\left(N^{*}(x, \Lambda) \geqq 1\right)\right) \\
& =|\Lambda|^{-1} \sum_{x \in \Lambda} E\left(1-N^{*}(x, \Lambda)\right) .
\end{aligned}
$$

Although neither $f_{A}^{*}$ nor $f$ need be convex, (5.5) leads to the next proposition which shows that they can be made convex by a slight alteration (see also [DN]). We define

$$
N^{*}(x)=\lim _{\Lambda \uparrow \mathbb{Z}^{d}} N^{*}(x, \Lambda)
$$

$N^{0}(x)$ is the number of distinct clusters to which the (occupied) neighbors of $x$ belong, after $x$ is set to be vacant. $N^{+}(x)$ is the same except that all infinite clusters are identified; i.e.

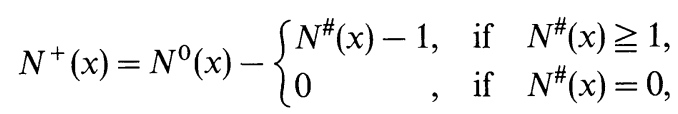

where $N^{\#}(x)$ is the number of distinct infinite clusters to which the (occupied) neighbors of $x$ belong, after $x$ is set to be vacant.

The next proposition is the analogue of Proposition 1.4.

Proposition 5.1. In the site percolation model on $\mathbb{Z}^{d}$.

(a) $f(p)-2 d\left(p-p^{2} / 2\right)$ is a convex function of $p$ so that $f$ has finite one-sided derivatives for all $p$ in $(0,1)$. These are given by

$$
\frac{\partial f}{\partial p}(p-0)=1-E\left(N^{0}(0)\right)
$$

$$
\frac{\partial f}{\partial p}(p+0)=1-E\left(N^{+}(0)\right)
$$

Thus $\partial f / \partial p$ has a discontinuity at some $p$ in $(0,1)$ if and only if for that value of $p$,

$$
E\left(\left[N^{\#}(0)-1\right] I\left(N^{\#}(0) \geqq 1\right)\right)>0 .
$$

Proof. Let us define

$V(x, \Lambda)=$ number of nearest neighbors of $x$ which are in $\Lambda$ and vacant.

For any $x$ in $\Lambda$, it is easy to see that $N^{*}(x, \Lambda)+V(x, \Lambda)$ cannot increase when a site is changed from vacant to occupied, hence

$$
E\left(N^{*}(x, \Lambda)+V(x, \Lambda)\right) \text { is decreasing in } p .
$$

Let us denote by $w(x, \Lambda)$ the number of nearest neighbors of $x$ which belong to $\Lambda$; $w(x, \Lambda)=2 d$ for every $x$ in $\Lambda$ not near the boundary of $\Lambda$. Since

$$
E(V(x, \Lambda))=w(x, \Lambda)(1-p)=\frac{d}{d p}\left[w(x, \Lambda)\left(p-p^{2} / 2\right)\right]
$$


it follows from (5.5) and (5.9) that

$$
\tilde{f}_{\Lambda}^{*}(p) \equiv f_{\Lambda}^{*}(p)-|\Lambda|^{-1} \sum_{x \in \Lambda}\left\{w(x, \Lambda)\left(p-p^{2} / 2\right)\right\}
$$

is a convex function of $p$.

It is clear that as $\Lambda_{k} \rightarrow \mathbb{Z}^{d}$, e.g. in the sense of Lemma 2.1,

$$
f_{\Lambda_{k}}^{*}-\tilde{f}_{\Lambda_{k}}^{*} \rightarrow 2 d\left(p-p^{2} / 2\right)
$$

Since, as mentioned above, Lemma 2.1 is valid for site percolation, we have

$$
\tilde{f}_{\Lambda_{k}}^{*}(p) \rightarrow f(p)-2 d\left(p-p^{2} / 2\right)
$$

which yields statement a) of the proposition. The remainder of the proof is based on essentially the same arguments as in the proofs of Lemmas 2.3 and Proposition 1.4 given in Sect. 2. For example, the one sided continuity of $\underset{k}{\lim } \partial f_{A_{k}}^{*} / \partial p$ is based on the fact that the expectation in (5.9) is monotonic in $\Lambda$ (for $x$ in $\Lambda$, not near the boundary of $\Lambda$ ). To avoid repetition, further details are left to the reader.

The next proposition is the analogue of Proposition 1.5. We do not present its proof since it is essentially the same as the one give in Sect. 3 (e.g. for the nearest neighbor bond model) but easier since (3.1) and (3.4) are simplified to

$$
P_{n}=\sum_{l} a_{n l} p^{n}(1-p)^{l}
$$

where $a_{n l}$ is the number of site lattice animals with $n$ occupied (volume) sites and $l$ vacant (surface) sites.

Proposition 5.2. In the site percolation model on $\mathbb{Z}^{d}$, the free energy $f$ is a continuously differentiable function of $p$ in $(0,1)$.

Remark. Extensions to site percolation of Proposition 3.3 and its three corollaries are valid. These concern twice-differentiability of $f$ at $p_{c}$ and inequalities for the critical exponent $\gamma$. The proofs are again essentially the same as for bond models.

The next proposition is the analogue of Proposition 1.1.

Proposition 5.3. In the site percolation model on $\mathbb{Z}^{d}$, for any $p$ in $[0,1]$ either there is no infinite cluster with probability one or else there is exactly one infinite cluster with probability one.

Proof. The proposition is trivial for $p=0$ or 1 . If $0<p<1$, then Propositions 5.1 and 5.2 imply that the expectation in (5.8) is always zero and thus that

$$
\operatorname{Prob}\left(N^{\#}(0) \geqq 2\right)=0 \text {. }
$$

To prove Proposition 5.3 it suffices to assume that more than one infinite cluster occurs with nonzero probability and derive a contradiction to (5.13). Under this assumption, $\operatorname{Prob}(G(\Lambda))$ is positive for some large cube $\Lambda$ centered at the origin, where $G(\Lambda)$ is the event that (at least) two distinct infinite clusters occur and intersect $\Lambda$. It is easy to construct, as in [NS1], a mapping $\Phi$ on configurations $\omega$ in $G(\Lambda)$ which only alters the occupation status of sites in $\Lambda$ and for which $\Phi(G(\Lambda))$ is contained in the event that $N^{\#}(0) \geqq 2$. But, as in the proof of Proposition 1.1 given in 
Sect. 4, $\operatorname{Prob}(G(\Lambda))>0$ implies $\operatorname{Prob}(\Phi(G(\Lambda)))>0$ implies $\operatorname{Prob}\left(N^{\sharp}(0) \geqq 2\right)>0$ which contradicts (5.13).

The next proposition combines the analogues of Proposition 1.2 and Proposition 1.3. Continuity of the connectivity functions is proven as for bond models; continuity of $P_{\infty}$ is an immediate consequence of Proposition 5.3 and the main result of van den Berg and Keane [BK].

Proposition 5.4. In the site percolation model on $\mathbb{Z}^{d}, \tau\left(x_{1}, \ldots, x_{n}\right)$ is a continuous function of $p$ for all $p$ and $P_{\infty}$ is a continuous function of $p$ except possibly at $p_{c}$.

Remark. $P_{\infty}$ for standard site models with $d \geqq 2$ should be continuous also at $p_{c}$. This has not yet been rigorously proven except for $d=2$ [Rus].

We conclude this section and the paper with a brief discussion of the free energy for mixed site-bond models which shows that all our results, suitably formulated, are valid for these models. Site-bond models have their own intrinsic interest and in addition they arise naturally when analyzing, e.g., pure bond models by block renormalization techniques, as in [ACCFR; NS2]. In such a model (on $\mathbb{Z}^{d}$ ) bonds $\{x, y\}$ are occupied with probability $p_{y-x}$ and sites are occupied (or "alive") with probability $\lambda$, all independently. Denoting by $p$ the common value of $p_{y-x}$ for $y-x$ in some $\Gamma$, as in a pure bond model, we may consider a one-parameter family of such models using either the parameter $\lambda$ or $p$ (or $\beta$ ).

$C(x)$, the (site) cluster of $x$, consists of all occupied sites which are connected to $x$ by paths of occupied bonds both of whose endpoints are occupied; $C(x)$ is empty if $x$ is vacant. As usual $P_{n}=\operatorname{Prob}(|C(0)|=n)$ for $n=0,1, \ldots, \infty$, and the free energy is

$$
f=\sum_{n \geqq 1} n^{-1} P_{n}
$$

which represents the expected number of nonempty clusters per site.

The convexity properties of $f$ (and its finite volume approximations), as a function of $p$ or $\beta$, are the same as in a pure bond model. Convexity as a function of $\lambda$ is essentially the same as for a pure site model except that here the quantity $N^{0}(x)$ is redefined as the number of distinct clusters to which those occupied sites $y$, such that $\{x, y\}$ is also occupied, belong after $x$ is set to be vacant. $N^{+}(x), N^{*}(x, \Lambda)$ etc. are defined similarly. Analogues of Propositions 1.4 and 5.1 can then be shown by arguments like those used above.

The analogues of Propositions 1.5 and 5.2 are also obtained by similar arguments to those used above. The lattice animal expansion (jointly in $\lambda$ and $p$ ) of $P_{n}$ has the form

$$
P_{n}=\sum_{l^{\prime}, m, l} a_{n l^{\prime} m l} \lambda^{n}(1-\lambda)^{l^{\prime}} p^{m}(1-p)^{l},
$$

where $a_{n l^{\prime} m l}$ depends on the $p_{z}$ 's for $z$ not in $\Gamma$. One then first sums over $m, l$ or over $l^{\prime}$, before applying the arguments like those of Sect. 3. Finally one obtains analogues of Proposition 1.1 and Proposition 5.3 and of Propositions 1.2-1.3 and Proposition 5.4 by analogous arguments. 
We wish to thank S. Orey, G. Sell and H. Weinberger for their hospitality. Two of us (M. A. and C. M. N.) also thank the John S. Guggenheim Foundation for its fellowhip support.

\section{References}

[AB1] Aizenman, M., Barsky, D. J.: Sharpness of the phase transition in percolation models, Commun. Math. Phys. 108, 489-526 (1987).

[AB2] Aizenman, M., Barsky, D. J.: in preparation. See also Barsky, D. J. Rutgers University Ph.D. thesis (1987).

[ACCFR] Aizenman, M., Chayes, J. T., Chayes, L., Fröhlich, J., Russo, L.: On a sharp transition from area law to perimeter law in a system of random surfaces. Commun. Math. Phys. 92, 19-69 (1983)

[AN1] Aizenman, M., Newman, C. M.: Tree graph inequalities and critical behavior in percolation models. J. Stat. Phys. 36, 107-143 (1984)

[AN2] Aizenman, M., Newman, C. M.: Discontinuity of the percolation density in one-dimensional $1 /|x-y|^{2}$ percolation models. Commun. Math. Phys. 107, 611-648 (1986)

[BK] van den Berg, J., Keane, M.: On the continuity of the percolation probability function, Contemp. Math. 26, 61-65 (1984)

[BL] Bricmont, J., Lebowitz, J. L.: On the continuity of the magnetization and energy in Ising ferromagnets. J. Stat. Phys. 42, 861-869 (1986)

[C] Choquet, G.: Topology. New York: Academic Press 1966

[Co] Coniglio, A.: Shapes, surfaces, and interfaces in percolation clusters. In: Physics of finely divided matter. Daoud M. (ed.). Proc. Les Houches Conf. of March, 1985 (to appear)

[CCN] Chayes, J. T., Chayes, L., Newman, C. M., The stochastic geometry of invasion percolation. Commun. Math. Phys. 101, 383-407 (1985)

[DN] Durrett, R., Nguyen, B.: Thermodynamic inequalities for percolation. Commun. Math. Phys. 99, 253-269 (1985)

[F] Fisher, M. E.: Critical probabilities for cluster size and percolation problems. J. Math. Phys. 2, 620-627 (1961)

[FK] Fortuin, C., Kastelyn, P.: On the random-cluster model I. Introduction and relation to other models. Physica 57, 536-564 (1972)

[FKG] Fortuin, C., Kastelyn, P., Ginibre, J.: Correlation inequalities on some partially ordered sets. Commun. Math. Phys. 22, 89-103 (1971)

[G1] Grimmett, G. R.: On the number of clusters in the percolation model. J. Lond. Math. Soc. (2) 13, 346-350 (1976)

[G2] Grimmett, G. R.: On the differentiability of the number of clusters per vertex in the percolation model. J. Lond. Math. Soc. (2) 23, 372-384 (1981)

[GKM] Grimmett, G. R., Keane, M., Marstrand, J. M.: On the connectedness of a random graph. Math. Proc. Comb. Philos. Soc. 96, 151-166 (1984)

[H1] Hammersley, J. M.: Percolation processes. Lower bounds for the critical probability. Ann. Math. Statist. 28, 790-795 (1957)

[H2] Hammersley, J. M.: A Monte Carlo solution of percolation in the cubic crystal. Meth. Comp. Phys. 1, 281-298 (1963)

[Ha] Hankey, A.: Three properties of the infinite cluster in percolation theory. J. Phys. A 11, L49L55 (1978)

[Har] Harris, T. E.: A lower bound for the critical probability in a certain percolation process. Proc. Camb. Philos. Soc. 56, 13-20 (1960)

[K1] Kesten, H.: Percolation theory for mathematicians. Boston: Birkhäuser 1982

[K2] Kesten, H.: The incipient infinite cluster in two-dimensional percolation. Theor. Probab. Rel. Fields, 73, 369-394 (1986)

[K3] Kesten, H.: A scaling relation at criticality for 2D-percolation. In: Percolation theory and ergodic theory of infinite particle systems Kesten, H., (ed.). IMA volumes in mathematics and its applications, Vol. 8, Berlin, Heidelberg, New York: Springer (to appear)

[Ki] Kikuchi, R.: Concept of the long-range order in percolation problems, J. Chem. Phys. 53, 2713-2718 (1970) 
[KF] Kastelyn, P. W., Fortuin, C. M.: Phase transitions in lattice systems with random local properties. J. Phys. Soc. Jpn. 26, [Suppl], 11-14 (1969)

[KSh] Klein, S. T., Shamir, E.: An algorithmic method for studying percolation clusters. Stanford Univ. Dept. of Computer Science, Report no. STAN-CS-82-933 (1982)

[KSo] Kunz, H., Souillard, B.: Essential singularity in percolation problems and asymptotic behavior of cluster size distribution. J. Stat. Phys. 19, 77-106 (1978)

[L1] Leath, P. L.: Cluster shape and critical exponents near percolation threshold. Phys. Rev. Lett. 36, 921-924 (1976)

[L2] Leath, P. L.: Cluster shape and boundary distribution near percolation threshold. Phys. Rev. B 14, 5046-5055 (1976)

[Le] Lebowitz, J. L.: Coexistence of phases in Ising ferromagnets. J. Stat. Phys. 16, 463-476 (1977)

[N1] Newman, C. M.: In equalities for $\gamma$ and related critical exponents in short and long range percolation. In: Percolation theory and ergodic theory of infinite particle systems Kesten, H., (ed.). IMA volumes in mathematics and its applications, Vol 8. Berlin, Heidelberg, New York: Springer (to appear)

[N2] Newman, C. M.: Some critical exponent inequalities for percolation. J. Stat. Phys. 45, 359368 (1986)

[NS1] Newman, C. M., Schulman, L. S.: Infinite clusters in percolation models. J. Stat. Phys. 26, 613-628 (1981)

[NS2] Newman, C. M., Schulman, L. S.: One-dimensional $1 /|j-i|^{s}$ percolation models: The existence of a transition for $s \leqq 2$. Commun. Math. Phys. 104, 547-571 (1986)

[PS] Pike, R., Stanley, H. E.: Order propagation near the percolation threshold. J. Phys. A 14, L169-L177 (1981)

[R] Rockafellar, T. R.: Convex analysis. Princeton, NJ: Princeton Univ. Press 1970

[Ru] Ruelle, D.: Statistical mechanics: Rigorous results. New York: W. A. Benjamin 1969

[Rus] Russo, L.: On the critical percolation probabilities. Z. Wahrscheinlichkeitstheor Verw. Geb. 56, 229-237 (1981)

[SE] Sykes, M. F., Essam, J. W.: Exact critical percolation probabilities for site and bond problems in two dimensions. J. Math. Phys. 5, 1117-1127 (1964)

[W] Wierman, J. C.: On critical probabilities in percolation theory. J. Math. Phys. 19, 1979-1982 (1978)

Communicated by M. E. Fisher

Received September 27, 1986; in revised form February 19, 1987

Note added in proof. Uniqueness of the infinite cluster in some dependent percolation models was recently proved by Gandolfi, Keane and Russo. 
